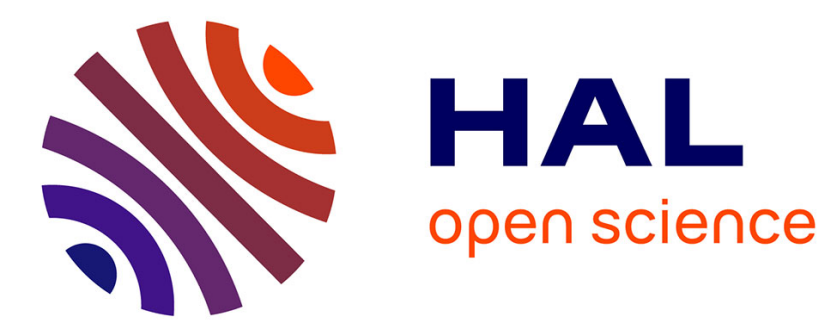

\title{
OSQAR chameleon afterglow search experiment
}

\author{
M. Sulc, P. Pugnat, R. Ballou, G. Deferne, J. Hosek, S. Kunc, A. Siemko
}

\section{To cite this version:}

M. Sulc, P. Pugnat, R. Ballou, G. Deferne, J. Hosek, et al.. OSQAR chameleon afterglow search experiment. Nuclear Instruments and Methods in Physics Research Section A: Accelerators, Spectrometers, Detectors and Associated Equipment, 2019, 936, pp.187-188. 10.1016/j.nima.2018.11.065 . hal-01991788

\section{HAL Id: hal-01991788 \\ https://hal.science/hal-01991788}

Submitted on 23 Nov 2020

HAL is a multi-disciplinary open access archive for the deposit and dissemination of scientific research documents, whether they are published or not. The documents may come from teaching and research institutions in France or abroad, or from public or private research centers.
L'archive ouverte pluridisciplinaire HAL, est destinée au dépôt et à la diffusion de documents scientifiques de niveau recherche, publiés ou non, émanant des établissements d'enseignement et de recherche français ou étrangers, des laboratoires publics ou privés. 


\title{
NIMA POST-PROCESS BANNER TO BE REMOVED AFTER FINAL ACCEPTANCE
}

\section{OSQAR chameleon afterglow search experiment}

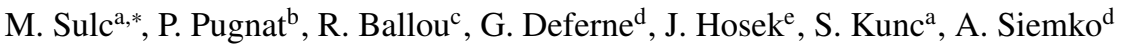 \\ ${ }^{a}$ Technical University of Liberec, Liberec, Czech Republic \\ ${ }^{b}$ LNCMI, CNRS \& Université Grenoble-Alpes, Grenoble, France \\ ${ }^{c}$ Institut Néel, CNRS \& Université Grenoble-Alpes, Grenoble, France \\ ${ }^{d}$ CERN, Geneva, Switzerland \\ ${ }^{e}$ Czech Technical University, Praha, Czech Republic
}

\begin{abstract}
OSQAR experiment has been extended to the quest of chameleon particles with environment-dependent mass from the search of a magnetic afterglow effect. OSQAR-CHASE (chameleon afterglow search) has been run in 2017 using one spare LHC dipole providing a $9 \mathrm{~T}$ transverse magnetic field with an $18.5 \mathrm{~W}$ laser and state-of-the art CCD detector. Experimental results with and without magnetic field were preliminary analyzed and no clear evidence for a photon-coupled chameleons was confirmed. The very weak false signals was observed with decay time $4300 \mathrm{~s}$. It can be explained by decrease of dark current from initial higher values during CCD cooling in detection phase. Increasing of the sensitivity of the OSQAR-CHASE experiment over the previous GammeV reference experiment assumes that the present coupling constant limit can be reduced around factor of 4 .
\end{abstract}

Keywords: Chameleon particle, Magneto-optical experiment, LHC dipole magnet

PACS: 12.60.Cn, 14.70.Bh, 14.80.Va, 95.36.+x

\section{Introduction}

The existence of dark energy, confirmed by astrophysical observation, can be explained by chameleon theories [1]. These scalar-tensor theories describe chameleons as particles with effective mass growing sharply at the proximity of higher density matter [2]. The chameleons can couple with photons in the presence of an external magnetic field. This field allows photon oscillation into chameleon and back again. The chameleons can manifest through an afterglow from the vacuum as they change to photons. This effect can be studied by magneto-optical laboratory experiments [3].

The first chameleon search laser experiments GammeV and GammeV-CHASE were performed with one Tevatron dipole magnet at Fermilab [4][5][6]. The $3.5 \mathrm{~W}$ second harmonic (532 $\mathrm{nm}$ ) beam from Nd:YAG laser was shining through a vacuum chamber closed by transparent windows, placed inside a $6 \mathrm{~m}$ long magnet with a $5 \mathrm{~T}$ magnetic field. The production time was over 5 hours, the detection time was 1 hour, and the time

\footnotetext{
${ }^{*}$ Corresponding author

Email address: miroslav.sulc@cern.ch (M. Sulc)
}

interval between production and detection phases was longer than $300 \mathrm{~s}$. No afterglow signal, induced by chameleon regeneration, was observed in the $1 \mathrm{~Hz}$ range of sensitivity. Only a signal in the orange and red portion of the spectrum (about $650-700 \mathrm{~nm}$ ) with decay time around $130 \mathrm{~s}$ was observed. It also appeared when the magnetic field was off. Its exact origin is still unknown [7].

OSQAR-CHASE has been proposed to extend the pioneering work of the GammeV using a stronger $9 \mathrm{~T}$ magnetic field, a $14.3 \mathrm{~m}$ long magnets and more sensitive detection systems [8]. The quick switching system between production and detection phases was also applied to observe possible strong chameleonphoton interactions.

\section{OSQAR-CHASE experimental set-up}

The OSQAR-CHASE experiment has been performed at CERN. A single spare LHC dipole producing $9 \mathrm{~T}$ magnetic field over $14.3 \mathrm{~m}$ has been used. The dipole is connected to its cryogenic feedbox and vacuum pumps. The dipole apertures have been equipped with an anticryostat serving as vacuum chambers. The anticryostat was closed by two BK7 glass windows 
with antireflexion coating. This experimental set-up was very similar to GammeV. The system was tested at preparatory phase in 2016 and fine-tuned to experimental run in 2017. The Verdi V18 CW laser with maximal power $18.5 \mathrm{~W}$ was used in production phase as a source of $2.33 \mathrm{eV}(532 \mathrm{~nm})$ photons. The laser beam was extended by beam expander and collimated to reach the center of the output window. Both input and output parts of anticryostat are equipped by light-tight valves. The valves 1 and 2 on input and output are open at production phase whereas the valve 3 before detector is closed to protect the photon detector. On the contrary the valve 1 and 2 are light-tightly closed and the valve 3 is open during afterglow measurement. This valve system allows to switch very quickly (in 10-20 s) between production and afterglow phases. The optical focusing system, consisting from two converging lenses, has been optimized to bring a maximal number of photons from the anticryostat to the region of interest at detector. ROI was almost centered circle on the CCD with a diameter of about 540 pixels. A CCD detector was used for photon detection. The thermoelectric cooled CCD-Andor iKon-DU934P-BV is composed of an array of 1024 x 1024 square pixels of $13 \mu \mathrm{m}$ size with the used maximum binning of $16 \times 16$. The quantum efficiency, given by Andor iKon, is equal to $95 \%$ at $550 \mathrm{~nm}$. In comparison the sensitivity of the overall detection system was experimentally found to be $(0.65 \pm 0.03)$ detected events per one incoming photon. The readout noise measured at $50 \mathrm{kHz}$ is equal to 3.1 e/pixel for 16x16 binning whereas the dark current values were smaller than $0.00011 \mathrm{e} / \mathrm{pixel} / \mathrm{s}$ at $90^{\circ} \mathrm{C}$ for $16 \times 16$ binning.

\section{Preliminary experimental results of OSQAR-CHASE}

The set of runs was performed. Long runs consisted of 4 hours of production time followed by 4 hours of afterglow measurement and was divided to 14 intervals of $1000 \mathrm{~s}$ long CCD exposition time. The exponential decrease with starting amplitude above $4.3 \mathrm{~Hz}$ was observed. Decay time was $(4300 \pm 700) \mathrm{s}$ for all long runs, see figure1. But this signal was surprisingly higher outside ROI. The decay was inhomogeneous on the CCD image sensor. Detected signal count rate was lower inside the ROI than outside the ROI. It decreases more quickly in ROI. The difference between inside ROI and outside ROI signals was stabilized to $2.9 \pm 0.4 \mathrm{~Hz}$ after $10000 \mathrm{~s}$.

The origin of false signal with long decay time about $4300 \mathrm{~s}$ is under investigation. This long time leads to a hypotheses privileging thermal origin for this false signal. The $18 \mathrm{~W}$ laser was shining during 4 hours through the output window to the beam dumper. Although this dumper collects almost all the beam energy, only a small amount of this one can be accumulated by the output window and by some parts close to the CCD. Emission of thermal IR photons from these parts together with conduction of heat are in equilibrium after 4 hours of chameleon production and dark current distribution on CCD is homogeneous. The slow cooling starts after laser switching-off and opening of valve 3 . It can disturb this equilibrium especially on the Peltier cooled CCD chip. The dark current is more suppressed in the central region i.e in the ROI, where the cooling Peltier element is located. The exponential decay afterglow

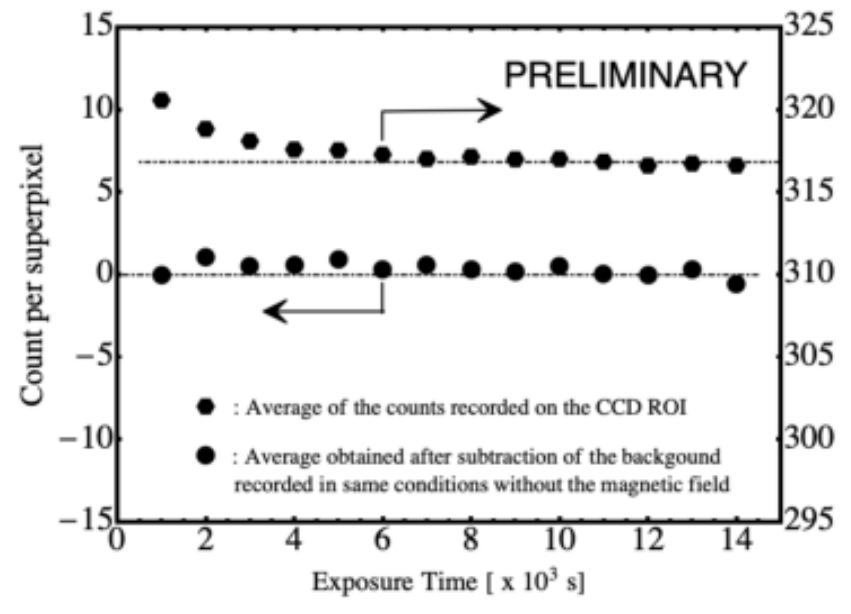

Figure 1: Preliminary results of the OSQAR-CHASE 2017 experimental run for scalar Chameleon search recorded with the magnetic field and after subtraction of the background determined without magnetic field.

signal was observed at all types of our runs. But it disappears once the difference between data taken with and without magnetic field is performed.

\section{Conclusion}

Experimental results were preliminary analyzed and no magnetic afterglow signal was observed at a frequency higher than $0.7 \mathrm{~Hz}$. Increasing of the sensitivity of the OSQAR-CHASE experiment over the previous GammeV reference experiment magnetic field from $5 \mathrm{~T}$ to $9 \mathrm{~T}$, power from $3.5 \mathrm{~W}$ to $18.5 \mathrm{~W}$, detector efficiency from 0.29 to 0.65 , decay rate sensitivity from $1.35 \mathrm{~Hz}$ to $0.65 \mathrm{~Hz}$ - assumes that the present coupling constant limit will be lowered by a factor of 4 .

\section{Acknowledgments}

This work was supported by the Ministry of Education, Youth and Sports of the Czech Republic by the Projects No. LM 2015058 and No. LTT17018.

\section{References}

[1] J. Khoury, and A. Weltman, Chameleon cosmology, Phys. Rev. D. 69 (2004) 044026.

[2] J. Khoury, and A. Weltman, Chameleon fields: awaiting surprises for tests of gravity in space, Phys. Rev. Lett. 93 (2004) 171104

[3] A. Upadhye, J. H. Steffen, and A. S. Chou, Designing dark energy afterglow experiments, Phys. Rev. D 86 (2012) 035006 .

[4] A. S. Chou, et al., Search for Chameleon Particles Using a PhotonRegeneration Technique, Phys. Rev. Lett. 102 (2009) 030402.

[5] A. Upadhye, J. H. Steffen, and A. Weltman, Constraining chameleon field theories using the GammeV afterglow experiments, Phys. Rev. D 81 (2010) 015013 .

[6] J. H. Steffen, et al., Laboratory Constraints on Chameleon Dark Energy and Power-Law Fields, Phys. Rev. Lett. 105 (2010) 261803 .

[7] J. H. Steffen, et al., Anomalous afterglow seen in a chameleon afterglow search, Phys. Rev. D. 86 (2012) 012003

[8] P. Pugnat, et al., OSQAR-CHASE Proposal, CERN-SPSC-2015010/SPSC-P-331-ADD-1, (2015) 